\title{
Decreased expression of hepatic cytochrome P450 1A2 (CYP1A2) in a chronic intermittent hypoxia mouse model
}

\author{
Xiao-Bin Zhang* ${ }^{*}$ Yi-Ming Zeng, Xiao-Yang Chen*, Yi-Xiang Zhang, Jin-Zhen Ding, Cheng Xue \\ Department of Respiratory and Critical Care Medicine, The Second Affiliated Hospital of Fujian Medical University, Center of Respiratory \\ Medicine of Fujian Province, Quanzhou 362000, China \\ Contributions: (I) Conception and design: YM Zeng, XY Chen; (II) Administrative support: YM Zeng, XY Chen; (III) Provision of study materials or \\ patients: JZ Ding, C Xue; (IV) Collection and assembly of data: XB Zhang, YX Zhang, C Xue, JZ Ding; (IV) Data analysis and interpretation: XB \\ Zhang, JZ Ding; (V) Manuscript writing: All authors; Final approval of manuscript: All authors. \\ *These authors contributed equally to this work. \\ Correspondence to: Yi-Ming Zeng. Department of Respiratory and Critical Care Medicine, The Second Affiliated Hospital of Fujian Medical \\ University, Center of Respiratory Medicine of Fujian Province, No. 34, Zhongshanbei Road, Licheng District, Quanzhou 362000 , China. \\ Email: ymzeng08@163.com.
}

\begin{abstract}
Background: Hepatic cytochrome P450 (CYP) isoforms, CYP1A2, is one of important enzymes for many drugs metabolism. Studies have confirmed that sustained hypoxia can influence the expression of hepatic CYP, including CYP1A2. The impact of chronic intermittent hypoxia (CIH), a marked characteristic of sleep apnea, on CYP1A2 remains unclear. The aim of the present study was to evaluate the effect of CIH on the expression of hepatic CYP1A2 in a mouse model with sleep apnea.

Methods: Twenty four old male (6-8 weeks) C57BL/6J mice ( $\mathrm{n}=12$ in each group) were randomly assigned to either normoxia group or CIH group. Mice in CIH group underwent 12 weeks intermittent hypoxia exposure. The different gene expression of hepatic CYP1A2 between two groups was analyzed by quantity real-time polymerase chain reaction. The protein levels of hepatic CYP1A2 in each group were observed by using western blotting and immunohistochemistry.

Results: After 12 weeks of exposure to intermittent hypoxia, the expression of hepatic CYP1A2, at the mRNA and protein levels was decreased more significantly in the CIH group than the normoxia group $(\mathrm{P}<0.01)$.

Conclusions: CIH contributes to inhibiting the expression of hepatic CYP1A2. This implies that the dosage of drugs metabolized by CYP1A2, should be adjusted in patients with sleep apnea.
\end{abstract}

Keywords: Intermittent hypoxia; hepatic cytochrome P450 (hepatic CYP); cytochrome P450 1A2 (CYP1A2);

sleep apnea; drug metabolism

Submitted Jul 26, 2017. Accepted for publication Dec 19, 2017.

doi: $10.21037 /$ jtd.2017.12.106

View this article at: http://dx.doi.org/10.21037/jtd.2017.12.106

\section{Introduction}

Cytochrome P450 1A2 (CYP1A2), one of the most important hepatic cytochrome P450 (CYP) isoforms, has been widely accepted to be an important drug for metabolic enzyme. Many drugs, such as theophylline, warfarin, and caffeine are metabolized by hepatic CYP1A2 $(1,2)$. Many factors can influence the activity of hepatic CYP. Hypoxia is generally a significant factor. Both human and animal studies have confirmed the impact of sustained hypoxia on CYP isoforms and relative drug biotransformation (3-6). Rahman and Thomas (3) have studies Altantic croaker and showed that hypoxia exposure causes a significant decrease in hepatic CYP1A mRNA and protein levels. Fradette et al. (4) found that after exposure to acute moderate hypoxia, hepatic CYP isoforms, CYP1A1, 1A2, 2B4, 2C5, and 2C16 
were down-regulated, while CYP3A6 and P-glycoprotein were up-regulated, $\mathrm{Li}$ et al. demonstrated that the activity of hepatic CYP family, CYP1A2 rather than CYP2C9 and CYP2C19, were decreased after rat were subjected to highaltitude hypoxia (5). A human study with 12 volunteers exposed to altitude-induced hypoxia found that the activity of CYP2D6 and CYP3A4 was slightly reduced (6).

Obstructive sleep apnea (OSA) is a highly prevalent disorder among middle-aged adult. OSA is associated with sleepiness, traffic accidents, and variety of diseases, such as hypertension, cardiovascular diseases, and metabolic abnormalities (7). Repetitive upper airway collapse during sleep in OSA patients induces chronic intermittent hypoxia $(\mathrm{CIH})$ and sleep fragmentation that are considered as the markedly pathophysiological features of OSA (7). Recently, clinical investigations have also elucidated that OSA is tightly correlated with liver damage or live disease (8-10). The severity of OSA is critically associated with elevated liver enzymes and steatohepatitis independent of the body weight (10). OSA patients have five-fold more risk to develop liver diseases (8). Continuous positive airway pressure (CPAP), the novel treatment choice for OSA, is indicated as a feasible way to decrease the risk of liver diseases (11). The repetitive obstruction of the upper airway contributes to nocturnal $\mathrm{CIH}$ and sleep fragmentation. Although the sleep fragmentation potentially results in OSA complications, the majority of experimental studies consider $\mathrm{CIH}$ as the major factor leading to oxidative stress, systemic inflammation, endothelial dysfunction, and the damage of several organs (12-14). Therefore, CIH animal model has been widely accepted as the model mimicking OSA patients $(15,16)$. In experimental studies, several studies paid attention to the correlation between $\mathrm{CIH}$ and liver injury or diseases. Ten year ago, evidence has shown CIH predisposed to mild liver injury in lean mice exposure to intermittent hypoxia for 12 weeks (17). A study by Savransky et al. (18) indicated that CIH combined with acetaminophen caused liver injury. The same team also inferred that CIH contributed to hepatitis in a mouse model of diet-induced fatty liver (19). We previous demonstrated that under the observation of transmission electron microscope, obvious pathological ultrastructural changes of liver cells were found in mice treated with $\mathrm{CIH}$ and isoniazid plus rifampicin. Another study from our team not only observed the ultrastructural liver cells changes, but also delineated that $\mathrm{CIH}$ down-regulated the total microsomal CYP protein levels (20). The metabolism of theophylline in hepatocytes harvested from mice was attenuated by $\mathrm{CIH}$ in our abovementioned study (20).

As mentioned above, hypoxia contributed to the change of CYP isoforms. The dosage of drugs may need to be regulated in patients with sustained hypoxia, such as chronic obstructive pulmonary disease, and chronic heart failure. The pathophysiological features of $\mathrm{CIH}$ are different to sustained hypoxia $(21,22)$. However, few data have been addressing the hepatic CYP levels and relative drug biotransformation in patients with OSA. Theophylline, as a probe drug to assess CYP1A2 activity, has been confirmed to be inhibited by $\mathrm{CIH}$ in our previous study (20). We also speculated that the expression of hepatic CYP1A2 appears to be decreased in $\mathrm{CIH}$-exposed mice. The aim of the present study was to evaluate the impact of $\mathrm{CIH}$ on hepatic CYP1A2 levels in a mouse model of sleep apnea, and to further elucidate the possible potential mechanisms for this effect.

\section{Methods}

\section{Animals}

A total of 24 wild-type, 6-8-week-old male, lean C57BL/6J mice were purchased from the Chinese Academy of Science Laboratory Animals Center in Shanghai, China. Experiments were implemented in accordance with the Guide for the Care and Use of Laboratory Animals (23). All the experimental procedures were approved by the Ethics Committee of The Second Affiliated Hospital of Fujian Medical University (Approved number 2016-008). All efforts had been made to minimize the number of mice used and their suffering.

\section{Experimental design}

All animals were in a controlled environment $\left(22-25^{\circ} \mathrm{C}\right.$ with a 12-12 h light-dark cycle; lights on at 08:00 am) where food and water were available. Mice were randomly allocated to two groups: normoxia group $(\mathrm{n}=12)$ and $\mathrm{CIH}$ group $(\mathrm{n}=12)$. Mice in CIH group were exposed to CIH condition; while mice in normoxia group were under normoxic condition during experiment. Both were produced for 12 weeks.

\section{CIH protocol}

CIH exposure was conducted as previously described (20,24,25), with some modification. Briefly, a self-made plexiglass chamber with one-way valves was served as $\mathrm{CIH}$ condition. The mice in the $\mathrm{CIH}$ group were 
Table 1 Primers for CYP isoforms RT-PCR

\begin{tabular}{llc}
\hline Primer name & Primers sequence(5' to 3') & $\begin{array}{c}\text { Product } \\
\text { length (bp) }\end{array}$ \\
\hline GAPDH & AAGGGCTCATGACCACAGTC & 20 \\
Abcb1a & TGACTATGCGAAAGCCACAG & 20 \\
CYP1A2 & CCCAAGGAGCGCTGTATCTA & 20 \\
CYP2A5 & CCCAAGGAGCGCTGTATCTA & 20 \\
CYP2C29 & GGAACATTGAGGACCGTGTT & 20 \\
CYP2E1 & AGGCTGTCAAGGAGGTGCTA & 20 \\
CYP3A11 & ACTTGAGGCAGAAGGCAAAG & 20 \\
CYP3A25 & GCATAGGCATGAGGTTTGCT & 20 \\
\hline
\end{tabular}

$\mathrm{CIH}$, chronic intermittent hypoxia; RT-PCR, real-time polymerase chain reaction; CYP, cytochrome P450.

placed in the chamber. A programmable instrument that regulated solenoid valves altered the input of nitrogen, oxygen, or compressed air to generate $\mathrm{CIH}$ condition. The S-450 oxygen detection alarm (IST-AIM Company, USA, with a sensitivity of $0.1 \%$ ) was used to monitor the oxygen concentration. The oxygen concentration in the chamber was altered from normal $(21 \% \pm 1 \%)$ to nadir point $(6 \% \pm 1 \%)$ for every $120 \mathrm{~s}$ of hypoxia and reoxygenation period. The $\mathrm{CIH}$ exposure was conducted from 08:00 am to 04:00 pm daily for 12 consecutive weeks.

\section{Samples collection and tissues preparation}

After the CIH exposure, mice were sacrificed for samples collection. Mice were fasted overnight prior to terminal anaesthesia. After being euthanized with 3\% phenobarbital $(30 \mathrm{mg} / \mathrm{kg})$ intraperitoneal injection, all mice were weighted. Blood was obtained by direct cardiac puncture. The blood samples were centrifuged at 3,000 g for $15 \mathrm{~min}$ at $4{ }^{\circ} \mathrm{C}$ while the supernatants (serum) were collected and stored at $-80{ }^{\circ} \mathrm{C}$ for further analysis. Liver was surgically isolated and weighted, either frozen in liquid nitrogen or then stored at $-80{ }^{\circ} \mathrm{C}$, fixed in buffered $10 \%$ formalin for histological examination, or immediately prepared for liver microsome collection.

\section{Analysis of alanine transaminase (ALT), aspartate transaminase (AST), and glutamyl transpeptidase (GGT)}

Serum ALT, AST and GGT were analyzed by using the H-7600 automatic analyzer (Hitachi, Japan) in the Clinical
Experimental Center at the Second Affiliated Hospital of Fujian Medical University.

\section{Quantity real-time polymerase chain reaction (qRT-PCR)}

Total RNA was extracted from mouse liver tissue using the TRIzol reagent (Invitrogen, Carlsbad, CA, USA). The concentration of total RNA was measured by spectrophotometry and the $\mathrm{OD}_{260} / \mathrm{OD}_{280}$ ratio was obtained to assess the RNA purity. To synthesize the first-strand complementary DNA (cDNA), reserve transcript was conducted with PrimeScript ${ }^{\mathrm{TM}}$ RT reagent Kit (TaKaRa, Biotechnology, Dalian, China) in a $20 \mu \mathrm{L}$ reaction mixture following the supplier's instructions. qRT-PCR was conducted using cDNA as a template in the Applied Biosystems 7,500 Fast Real-Time PCR System (Applied Biosystems, Foster City, CA, USA) with SYBR green I as fluorescent dye according to the protocol of manufacture (TaKaRa, Biotechnology, Dalian, China). The PCR conditions were $95^{\circ} \mathrm{C}$ for $30 \mathrm{~s}$, followed by 40 cycles of $95^{\circ} \mathrm{C} 3 \mathrm{~s}, 60^{\circ} \mathrm{C} 30 \mathrm{~s}$. The primers used in the RT-PCR are outlined in Table 1. The relative gene expression levels were calculated on the basis of $2^{\text {-DDCt }}$ GAPDH was used as internal control. The results are presented as fold change of $\mathrm{CIH}$ relative to normoxia group.

\section{Western blotting}

Liver tissue sections $(50-100 \mathrm{mg})$ were homogenized in a glass homogenizer with ice-cold radioimmunoprecipitation (RIPA) lysis buffer (500-1,000 $\mu \mathrm{L}$, Beyotime, Beijing, China), in which both phosphatase and protease inhibitors were added. All the procedures aforementioned were performed on ice. The homogenates were centrifuged at $12,000 \mathrm{~g}$, at $4{ }^{\circ} \mathrm{C}$ for $10 \mathrm{~min}$, therefore. The supernatants were extracted. Protein concentrations in the supernatants were determined by bicinchoninic acid protein assay (Beyotime, Beijing, China). Equal amounts of boiled protein (40 $\mu \mathrm{g}$ for each lane) in the loading buffer were separated on a $10 \%$ sodium dodecyl sulfate-polyacrylamide gel (SDS-PAGE), and transferred on to polyvinylidene difluoride (PVDF) membranes (Millipore, Boston, MA, USA). The membranes were blocked with $5 \%$ non-fat milk for at least $1 \mathrm{~h}$ at room temperature. After 3 times rinse with Tris-buffered saline + Tween-20 (TBST; $0.15 \mathrm{M} \mathrm{NaCl}$, $20 \mathrm{mM}$ Tris-HCl, $0.05 \%$ Tween 20, pH 7.4), the PVDF membranes were incubated with the following antibodies at $4{ }^{\circ} \mathrm{C}$ overnight: rabbit anti-CYP1A2 polyclonal antibody (1:1,000, Proteintech, Catalog No: 19936-1-AP), rabbit 
anti-CYP2E1 polyclonal antibody (1:1,000, Proteintech, Catalog No.: 19937-1-AP) mouse anti-HIF-1 $\alpha$ monoclonal antibody (1:250, Novus Biologicals, Littleton, CO), rabbit anti-NF- $\kappa$ B monoclonal antibody $(1: 1,000$, Cell Signaling Technology), rabbit anti-p44/42 MAPK monoclonal antibody (1:1,000, Cell Signaling Technology), rabbit antiphospho-p44/42 MAPK monoclonal antibody (1:2,000, Cell Signaling Technology), and rabbit anti-GAPDH polyclonal antibody (dilution 1:5,000, Proteintech). The following day, the membranes were rinsed with TBST before being incubated with goat anti-rabbi/mouse peroxidaseconjugated secondary antibody (Proteintech, Chicago, IL, USA) in room temperature for $1 \mathrm{~h}$. After the blots were washed three times with TBST buffer, they were developed and exposed using an enhanced chemiluminescence kit (Clarity $^{\mathrm{TM}}$ Western ECL Substrate, Bio-Rad). Western blots were performed triplicate for accuracy. The band densities on the membranes were estimated using the Image J analysis software (National Institutes of Health, Bethesda, MD, USA). GAPDH was used as an internal control to normalize sample loading on the gels.

\section{Histopathological examination and immunohistochemical analysis}

After overnight fixing in formalin, the liver tissues were dehydrated in graded alcohol series, cleaned with xylene, and embedded in paraffin. Coronal $4 \mu \mathrm{m}$ thick liver slices were cut. These slices were de-paraffinized with xylene, followed by rehydration in an ethanol gradient $(100-70 \% \mathrm{v} / \mathrm{v})$. Then these slices were stained with hematoxylin-eosin ( $\mathrm{HE})$. Immunohistochemistry staining was also conducted on the coronal slices of the tissue samples. Slices were rehydrated and rinsed in phosphatebuffered saline (PBS), and incubated in $1 \% \mathrm{H}_{2} \mathrm{O}_{2}$ for $30 \mathrm{~min}$ at room temperature and three rinses in PBS. Slides were blocked with 10\% goat serum (Solarbio, Beijing, China) for $15 \mathrm{~min}$ prior to be incubated with the primary antibody: rabbit anti-CYP1A2 (1:200, Proteintech) at $4{ }^{\circ} \mathrm{C}$ overnight. Subsequently, the slices were incubated in biotinylated goat anti-rabbit secondary antibody (1:500) for $1 \mathrm{~h}$ at room temperature. Avidin-3, 3'-diaminobenzidine (DAB) staining kit (Beijing Zhongshan Biotechnology, Beijing, China) was used to detect immune complexes. All slices were visualized using a Leica DM2500 microscope, and images were photographed at original magnification $40 \times, 100 \times$, respectively.

\section{Liver microsome preparation and CYP1A2 expression}

Liver microsome preparation was conducted as previous reported (20). Liver tissue about $250 \mathrm{mg}$ was rinsed with chill PBS, then homogenized in $0.05 \mathrm{~mol} / \mathrm{L} \mathrm{pH} \mathrm{7.4} \mathrm{Tris-HCL} \mathrm{buffer}$ solutions. Homogenate was centrifuged at $10,000 \mathrm{~g}$ for $20 \mathrm{~min}$ at $4{ }^{\circ} \mathrm{C}$. Supernatant was collected, and ultracentrifuged at $105,000 \mathrm{~g}$ for $60 \mathrm{~min}$ at $4{ }^{\circ} \mathrm{C}$ (Beckman, Optiam L-100XP, USA). Microsomal pellets were re-suspended in $0.05 \mathrm{~mL}$, $250 \mathrm{mM}$ sucrose, then flashed frozen and stored at $-80{ }^{\circ} \mathrm{C}$. Total CYP concentration in hepatic microsomes was measured with carbon monoxide difference spectroscopy (20). The microsomal protein level of CYP1A2 was also detected by western blotting as aforementioned.

\section{Statistical analysis}

The GraphPad Prism 5.0 (GraphPad software, Inc., La Jolla, CA, USA) was performed for data analysis. All values are presented as mean \pm standard deviation (SD). Independent-samples $t$-test was conducted to compare the differences of relative values between the two groups. A P value of $<0.05$ was considered statistically significant.

\section{Results}

\section{Body weight, liver weight, ALT and AST changes in mice} treated with $\mathrm{CIH}$

Compared to normoxia group, the body and liver weights of mice in CIH group were declined significantly $(21.86 \pm 1.60$ vs. $26.78 \pm 1.25,1.36 \pm 0.14$ vs. $1.50 \pm 0.18 ; \mathrm{P}<0.05, \mathrm{P}<0.01$; respectively) (Figure $1 A$ ). After 12 -week treatment with $\mathrm{CIH}$, the transaminases levels, namely ALT and AST, in CIH group were similar to the normoxia group $(34.58 \pm 12.77$

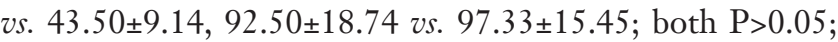
respectively) (Figure $1 B$ ).

\section{Liver histopatbology}

Histopathological examination exhibited that CIH did not affect the morphological architecture of live tissue in both $\mathrm{CIH}$ and normoxia groups on the $\mathrm{HE}$ stains (Figure 2).

\section{RT-PCR results of CYP isoforms $m R N A s$}

Figure 3 shows the RT-PCR results of hepatic CYP isoforms mRNAs. After exposure to CIH for 12 weeks, the CYP1A2 

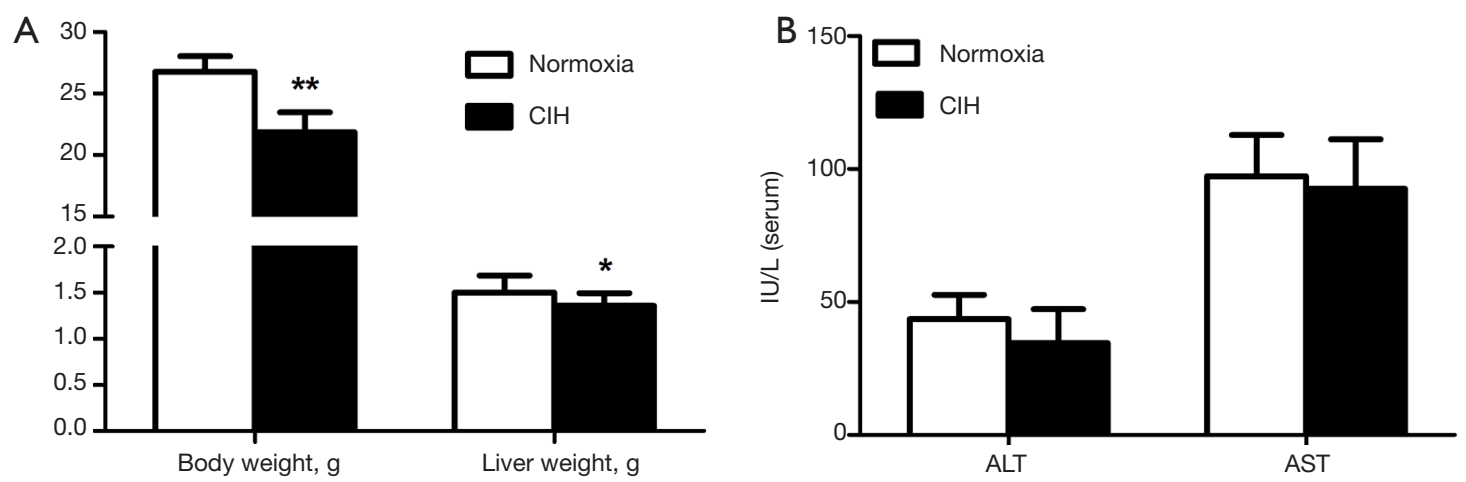

Figure 1 Comparison of (A) body weight and liver weight, (B) alanine transaminase (ALT) and aspartate transaminase (AST) in mice between normoxia and chronic intermittent hypoxia (CIH) groups. (A) In comparison with normoxia group, both body weight and liver weight were decreased in mice among CIH group. The difference of ALT and AST between two groups did not reach statistically significant. Data are presented as means \pm standard deviation $(\mathrm{SD}) .{ }^{*}, \mathrm{P}<0.05$ vs. normoxia group; ${ }^{* *}, \mathrm{P}<0.01$ vs. normoxia group.

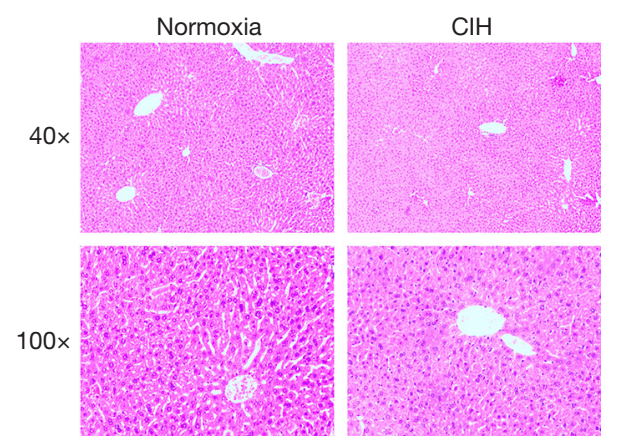

Figure 2 Histopathological examination of liver in mice subjected to normoxia and chronic intermittent hypoxia (CIH). No abnormal liver architecture change, namely inflammation, steatosis, hepatocyte swelling or necrosis, was detected under light microscope in both groups. The morphology of hepatic cells in two groups was detected by hematoxylin-eosin (HE).

mRNA levels were decreased but other CYP isoforms (Abcb1a, CYP2A5, CYP2C29, CYP2E1, CYP3A11, and CYP3A25) mRNAs were not altered.

\section{Western blotting results of CYP1A2 and CYP2E1}

Since the differential species between human and mouse, the CYP isoforms are also different. Both CYP1A2 and CYP2E1 are the hepatic CYP isoforms existing both in human and mouse. Hereby, the CYPA12 and CYP2E1 antibodies were applied in the present study. The results illustrated that the protein levels of CYP1A2 rather than CYP2E1 were decreased in CIH group (Figure 4).

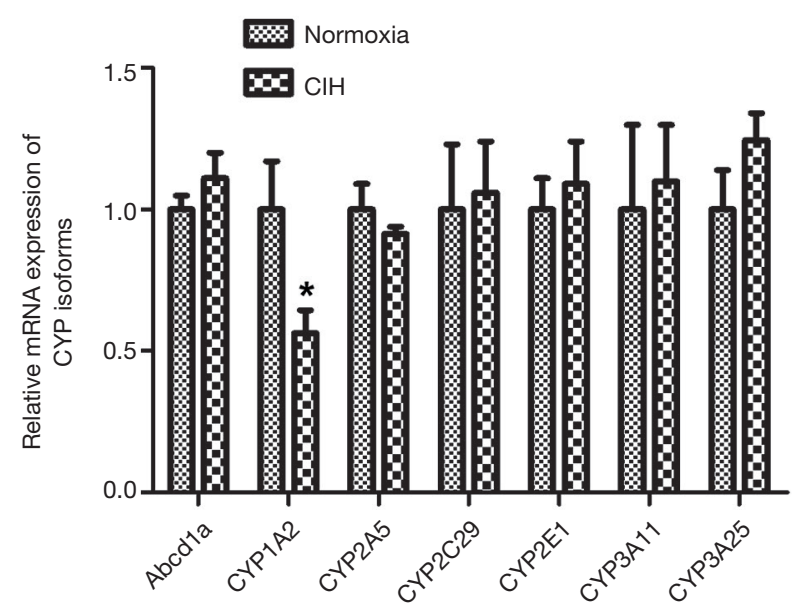

Figure 3 RT-PCR results of CYP isoforms mRNA. After 12 weeks of exposure to CIH, the mRNA levels of CYP1A2 were decreased, while the other CYP isoforms were not altered. *, $\mathrm{P}<0.05$ when compared with control group; CYP, cytochrome P450; CIH, chronic intermittent hypoxia; RT-PCR, real-time polymerase chain reaction.

\section{Expression of CYP1A2 both at mRNA and protein levels}

A reduction of total CYP450 concentration in hepatic microsomes was observed in mice subjected to $\mathrm{CIH}$ (Figure 5). The RT-PCR results revealed that CIH contributed to the down-regulation of CYP1A2 mRNA expression (Figure 6A). Immunohistochemistry assay presented that the liver tissue in mice exposure to $\mathrm{CIH}$ group presented a decreased expression of CYP1A2 when compared with mice in 


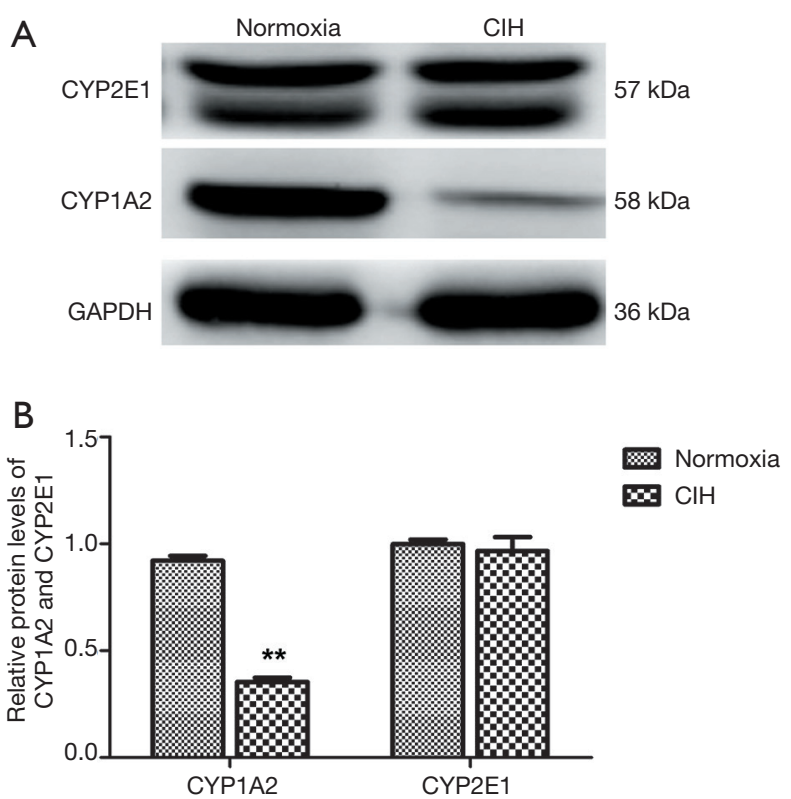

Figure 4 Western blotting results of CYP1A2 and CYP2E1. When compared with mice exposed to normoxia, the protein levels of hepatic CYP1A2 in the CIH group were decreased, however, the CYP2E1 protein levels remained no change in $\mathrm{CIH}$ group. **, $\mathrm{P}<0.01$ when compared with control group; CYP, cytochrome $\mathrm{P} 450$; CIH, chronic intermittent hypoxia.

normoxia group (Figure 6B). Immunohistochemistry results were confirmed by western blotting analysis: $\mathrm{CIH}$ decreased the CYP1A2 protein levels in both whole liver tissue fraction (Figure 6C) and liver microsomal fraction (Figure 6D).

\section{Protein levels of HIF-1a, NF-kB and p-P44/42 MAPK in mice treated with $\mathrm{CIH}$}

As shown in Figure 7, the CIH group displayed a significantly enhanced expression level of HIF-1 $\alpha$, NF- $\mathrm{kB}$, and p-P44/42 MAPK compared with normoxia group.

\section{Discussion}

The present study evaluated the impact of CIH on the expression of hepatic CYP1A2 levels in a mouse model of sleep apnea. The results showed that the expression of hepatic CYP1A2, both at the mRNA and protein levels, was inhibited in mice exposure to 12 -week CIH. Meanwhile, the protein levels of HIF- $1 \alpha$, NF- $\kappa B$, and p-P44/42 MAPK were also elevated in $\mathrm{CIH}$-exposed mice.

Most drugs should be metabolized by liver in the

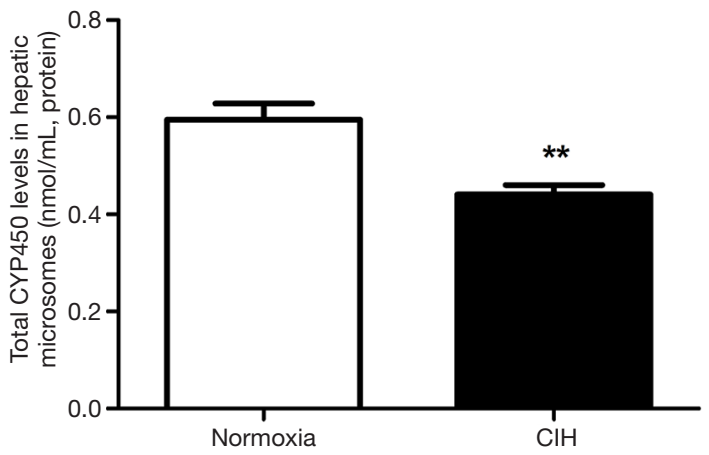

Figure 5 Total CYP450 levels of hepatic microsomes were lower in $\mathrm{CIH}$ group than that in normoxia group. ${ }^{* *}, \mathrm{P}<0.01$ vs. normoxia group; CYP, cytochrome $\mathrm{P} 450$; $\mathrm{CIH}$, chronic intermittent hypoxia.

body. CYP is a superfamily of heme-thiolate proteins. Numerous endogenous and exogenous compounds, including drugs, were metabolized by CYP enzymes (26). CYP could be subdivided to many isoforms; CYP1A2 is one of the major P450 isoforms, contributing nearly $20 \%$ of the hepatic P450 pool and catalyzing oxidative biotransformation of various types of commonly used drugs such as theophylline, caffeine, and clozapine $(1,2,27)$. The activity of hepatic CYP enzymes can be regulated by several endogenous and exogenous factors. Evidence elucidated that hypoxia is a significant factor contributing to the fluctuation of CYP expression (28). Several human studies have reported that chronic sustained hypoxia, which exists in chronic respiratory or heart failure, alters many drugs biotransformation $(29,30)$. Antipyrine is a drug eliminated exclusively by several hepatic CYP isoforms. Data confirmed that the clearance rate of antipyrine in patients with chronic obstructive lung disease is obviously lower than that in volunteers with normoxia (29). The clearance rate of theophylline, a probe drug evaluating the activity of CYP1A2, has been proven to be reduced by $75 \%$ in patients with severe respiratory failure (31). Animal study found that when rabbits exposed to a sustained hypoxia atmosphere for $48 \mathrm{~h}$, the hepatic CYP isoforms are prone to be modulated: CYP1 and CYP2 families were inhibited, while CYP3A6 and P-glycoprotein were enhanced (4). Chronic hypobaric hypoxia exposure not only down-regulated the expression of CYP2C9, but also affected the metabolism of ibuprofen in a rat model (32). Both acute and chronic high-altitude hypoxia elicited significant decrease in the expression of CYP1A2 in rats (5). The down-regulated CYP1A was observed in Atlantic 
A
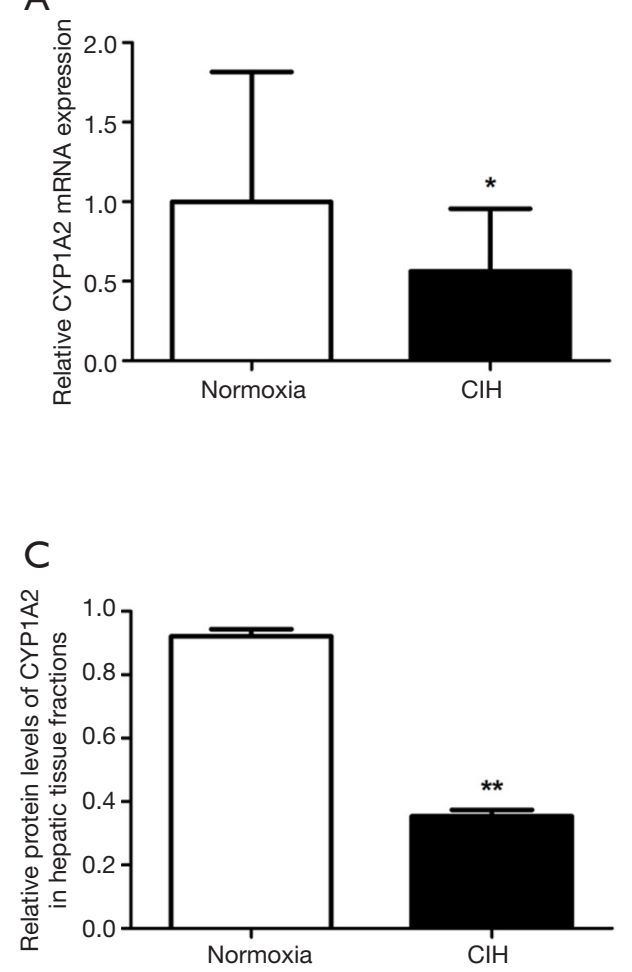

B
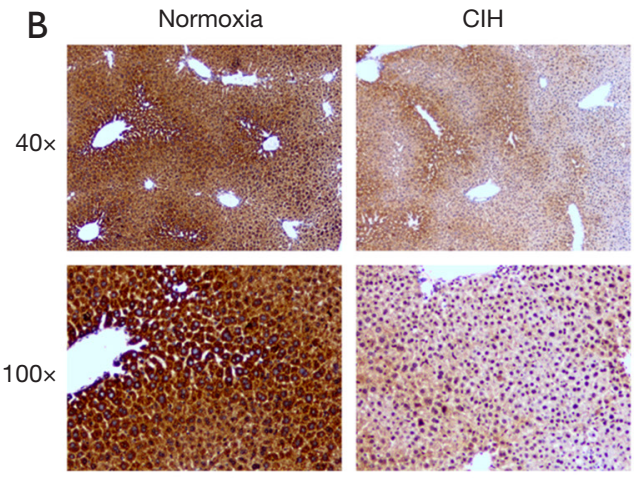

D

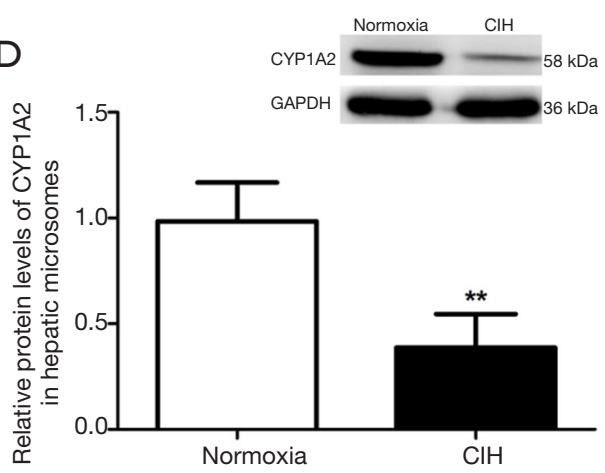

Figure 6 Chronic intermittent hypoxia (CIH) contributed to down-regulation of hepatic CYP1A2 expression. (A) RT-PCR results showed that in comparison with normoxia group, CYP1A2 mRNA levels were markedly inhibited in mice exposed to CIH. CYP1A2 in cytoplasm manifested as a brown color after immunohistochemical staining; (B) compared with normoxia group, immunochemistry assay illustrated a decreasing number of CYP1A2 immune-positive hepatocytes in CIH-induced mice. Western blotting results also confirmed that the protein levels of CYP1A2 were decreased in both whole (C) hepatic tissue fraction and (D) hepatic microsomal fraction among mice treated with CIH. GAPDH was served as loading control. Data are expression as means \pm standard deviation (SD). *, $\mathrm{P}<0.05$ vs. normoxia group; **, $\mathrm{P}<0.01$ vs. normoxia group; RT-PCR, real-time polymerase chain reaction.

croaker subjected to hypoxia (3). The down-regulation of CYP levels were also found in human hepatocytes under sever hypoxic incubation (33). From the knowledge abovementioned, it is not hard to conclude that hypoxia contributes to the up or down regulation of hepatic CYP. Since hypoxia reduces the clearance of selected drugs and increases drugs toxicity, the dosage of drugs which metabolized by CYP isoforms may need to be adjusted in clinical practice.

Robust evidence showed that OSA is a major independent risk factor for various liver diseases $(8,9,11)$. OSA patients suffered 5 -fold risk to develop liver cirrhosis and hepatitis $\mathrm{C}$ in a population-based study in Taiwan (8). A study on 163 nondrinking patients who underwent fullnight polysomnography showed that the severity of OSA is associated with elevated liver enzymes; Steatohepatitis and fibrosis were observed in histological examination in 18 patients with elevated liver enzymes (10). Meanwhile, study also delineated that the prevalence of OSA was up to $72 \%$ in patients with biopsy-proven nonalcoholic fatty liver disease (9). Experimental study demonstrated that $\mathrm{CIH}$ originated or promoted liver damage. Savransky and colleague (17) found that CIH caused high ALT in serum and hepatocytes swelling in histology examination among lean mice. The inflammation and apoptosis in the liver were exhibited in an animal model mimicking OSA $(19,34,35)$. The synergistic function of CIH and hepatotoxic drugs in the liver injury were described in previous studies $(18,25)$. Histopathological examination in a study by Savransky and co-workers (18) revealed hepatic necrosis and increased apoptosis in CIH-induced mice. Our previous study indicated that $\mathrm{CIH}$ aggravated hepatic 

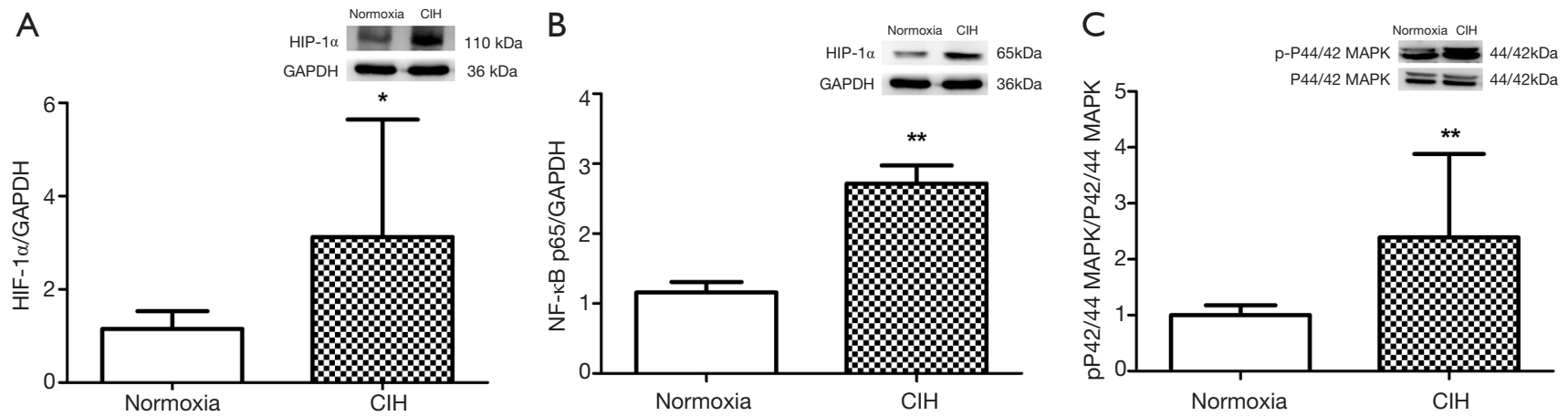

Figure 7 Western blotting results delineated that when compared with normoxia group, the protein expression levels of $\mathrm{HIF}-1 \alpha$, NF- $\kappa \mathrm{B}$ and p-P44/42 MAPK were enhanced in mice exposed to chronic intermittent hypoxia (CIH). GAPDH (in HIF-1 $\alpha, \mathrm{NF}-\kappa \mathrm{B}$ groups) and P44/42 MAPK (in p-P44/42 MAPK group) were served as loading control. *, $\mathrm{P}<0.05$ vs. normoxia group; **, $\mathrm{P}<0.01$ vs. normoxia group.

ultrastructure damage in mice administrated with two antituberculosis drugs (25). There were inconsistent results regarding the histopathological finding of liver tissue in mice exposure to CIH. Study from Savransky (18) and the present study failed to observe the morphological changes of liver tissue under the light microscope. In contrast, other studies $(17,34,35)$ illustrated various degrees of pathological changes in hepatocytes. We speculated that the $\mathrm{CIH}$ duration and discrepancy of study design may partly explain the inconsistency.

Inconsistent to sustained hypoxia, the pathophysiological characteristics of $\mathrm{CIH}$ is similar to ischemia/reperfusion injury (7). CIH easily triggers systemic inflammation, oxidative stress, endothelial damage, and metabolic abnormalities (7). Numerous data demonstrated the impact of sustained hypoxia on hepatic CYP and drug metabolism (3-5,32). Patients with chronic sustained hypoxia were advised to adjust the dosage of drugs (6). However, no clinical investigation addressed the drug metabolism in OSA patients, in which $\mathrm{CIH}$ is an essential feature. The evidence of $\mathrm{CIH}$-induced hepatic CYP expression is also lacking in animal study. Based on the hypothesis that $\mathrm{CIH}$ elicited the change of expression of hepatic CYP, several CYP isoforms in mouse were evaluated. CYP1A2, one of the vital isoforms of CYP, was depressed by CIH in both mRNA and protein levels, however, we fail to find any change of other CYP isoforms. Simultaneously, we try to explore the possible underlying molecular mechanism of the inhibited effect. The enhanced expression of HIF- $1 \alpha$, NF- $\kappa \mathrm{B}$, and p-P44/42 MAPK was found in the mice treated with CIH. These findings were consistent with previous studies $(17,34,36-38)$.
However, we only can speculate these signaling molecules may involve in the effect of CIH on CYP isoform. Further study, such as RNA interference at the cellular levels, is warranted to confirm the definitely function of these molecules.

The present study had it strengths. First, the present study was the first study to investigate the relationship between CIH and hepatic CYP. Secondly, inconsistent with other studies $(18,21,34,36)$, the mice were exposed to intermittent hypoxia for total 12 weeks in our study. This implies that mice treated with $\mathrm{CIH}$, more approaching to the pathophysiological feature of sleep apnea patients. Third, lean C57BL6/J mice were applied for excluding the influence of obesity on hepatic CYP expression, since data delineated that hepatic CYP activity and drug biotransform could be affected by obesity $(39,40)$.

Several limitations in this study were clarified as follows: (I) we only found the low expression of hepatic CYP1A2 in a mouse model of CIH. In order to verify the hypothesis that OSA patients need to adjust the drug dosage and prevent the drug liver toxicity, study addressing on the change of blood probe drugs concentration is required in a larger animal model mimicking OSA or in OSA patients; (II) instead of CYP1A2, the other CYP isoforms were not altered after exposing to $\mathrm{CIH}$ in the present study. The conclusion of the present study might not be extrapolated to other CYP isoforms; (III) we only evaluated the effect of CIH, one of the pathophysiological features of OSA, on hepatic CYP. Sleep fragmentation, the other feature of OSA, was not mimicked in the present study. This weakens the clinical significance of this work. 


\section{Conclusions}

The present study demonstrated that CIH contributed to the inhibition the expression of hepatic CYP1A2. We speculate that the dosage of probe drugs, metabolizing by CYP1A2, appears to be adjusted in OSA patients.

\section{Acknowledgements}

We are grateful to Xiao-Zhen Wang from the center laboratory of Medical College, Xiamen University for liver microsome preparation with ultracentrifuge (Beckman, Optiam L-100XP, USA).

Funding: This works was supported by grant 2015J01445 and 2018J01393 for Fund from Natural Science Foundation of Fujian Province, grant 2014JQNJC20 for Young people training project from Fujian Province Health System, and grant 2013Z55 for Fund from Quanzhou Science and Technology Bureau.

\section{Footnote}

Conflicts of Interest: The authors have no conflicts of interest to declare.

Ethical Statement: All procedures performed in studies involving animals were in accordance with the ethical standards of the institution or practice at which the studies were conducted.

\section{References}

1. Wojcikowski J, Daniel WA. Perazine at therapeutic drug concentrations inhibits human cytochrome $\mathrm{P} 450$ isoenzyme 1A2 (CYP1A2) and caffeine metabolism--an in vitro study. Pharmacol Rep 2009;61:851-8.

2. Plowchalk DR, Rowland Yeo K. Prediction of drug clearance in a smoking population: modeling the impact of variable cigarette consumption on the induction of CYP1A2. Eur J Clin Pharmacol 2012;68:951-60.

3. Rahman MS, Thomas P. Effects of hypoxia exposure on hepatic cytochrome P450 1A (CYP1A) expression in Atlantic croaker: molecular mechanisms of CYP1A downregulation. PLoS One 2012; 7:e40825.

4. Fradette C, Batonga J, Teng S, et al. Animal models of acute moderate hypoxia are associated with a downregulation of CYP1A1, 1A2, 2B4, 2C5, and 2C16 and upregulation of CYP3A6 and P-glycoprotein in liver. Drug
Metab Dispos 2007;35:765-71.

5. Li X, Wang X, Li Y, et al. Effect of exposure to acute and chronic high-altitude hypoxia on the activity and expression of CYP1A2, CYP2D6, CYP2C9, CYP2C19 and NAT2 in rats. Pharmacology 2014;93:76-83.

6. Jurgens G, Christensen HR, Brosen K, et al. Acute hypoxia and cytochrome P450-mediated hepatic drug metabolism in humans. Clin Pharmacol Ther 2002;71:214-20.

7. Jordan AS, McSharry DG, Malhotra A. Adult obstructive sleep apnoea. Lancet 2014;383:736-47.

8. Chou TC, Liang WM, Wang CB, et al. Obstructive sleep apnea is associated with liver disease: a population-based cohort study. Sleep Med 2015;16:955-60.

9. Petta S, Marrone O, Torres D, et al. Obstructive Sleep Apnea Is Associated with Liver Damage and Atherosclerosis in Patients with Non-Alcoholic Fatty Liver Disease. PLoS One 2015;10:e0142210.

10. Tanne F, Gagnadoux F, Chazouilleres O, et al. Chronic liver injury during obstructive sleep apnea. Hepatology 2005;41:1290-6.

11. Hang LW, Chen CF, Wang CB, et al. The association between continuous positive airway pressure therapy and liver disease development in obstructive sleep apnea/ hypopnea syndrome patients: a nationwide populationbased cohort study in Taiwan. Sleep Breath 2017;21:461-7.

12. Dewan NA, Nieto FJ, Somers VK. Intermittent hypoxemia and OSA: implications for comorbidities. Chest 2015;147:266-74.

13. Garvey JF, Taylor CT, McNicholas WT. Cardiovascular disease in obstructive sleep apnoea syndrome: the role of intermittent hypoxia and inflammation. Eur Respir J 2009;33:1195-205.

14. DeMartino T, Ghoul RE, Wang L, et al. Oxidative Stress and Inflammation Differentially Elevated in Objective Versus Habitual Subjective Reduced Sleep Duration in Obstructive Sleep Apnea. Sleep 2016;39:1361-9.

15. Veasey SC, Davis CW, Fenik P, et al. Long-term intermittent hypoxia in mice: protracted hypersomnolence with oxidative injury to sleep-wake brain regions. Sleep 2004;27:194-201.

16. Row BW, Liu $\mathrm{R}, \mathrm{Xu} W$, et al. Intermittent hypoxia is associated with oxidative stress and spatial learning deficits in the rat. Am J Respir Crit Care Med 2003;167:1548-53.

17. Savransky V, Nanayakkara A, Vivero A, et al. Chronic intermittent hypoxia predisposes to liver injury. Hepatology 2007;45:1007-13.

18. Savransky V, Reinke C, Jun J, et al. Chronic intermittent hypoxia and acetaminophen induce synergistic liver injury 
in mice. Exp Physiol 2009;94:228-39.

19. Savransky V, Bevans S, Nanayakkara A, et al. Chronic intermittent hypoxia causes hepatitis in a mouse model of diet-induced fatty liver. Am J Physiol Gastrointest Liver Physiol 2007;293:G871-7.

20. Chen XY, Zeng YM, Zhang YX, et al. Effect of chronic intermittent hypoxia on theophylline metabolism in mouse liver. Chin Med J (Engl) 2013;126:118-23.

21. Gozal D, Gileles-Hillel A, Cortese R, et al. Visceral White Adipose Tissue after Chronic Intermittent and Sustained Hypoxia in Mice. Am J Respir Cell Mol Biol 2017;56:477-87.

22. $\mathrm{Wu}$ W, Dave NB, Yu G, et al. Network analysis of temporal effects of intermittent and sustained hypoxia on rat lungs. Physiol Genomics 2008;36:24-34.

23. The Guide for the Care and Use of Laboratory Animals. ILAR J 2016;57:NP.

24. Wang WY, Zeng YM, Chen XY, et al. Effect of Telmisartan on local cardiovascular oxidative stress in mouse under chronic intermittent hypoxia condition. Sleep Breath 2013;17:181-7.

25. Wu RH, Zeng YM, Chen XY. Intermittent hypoxia and isoniazid plus rifampicin affect hepatic ultrastructure in mice. Chin Med J (Engl) 2011;124:4034-8.

26. Olsen L, Oostenbrink C, Jorgensen FS. Prediction of cytochrome P450 mediated metabolism. Adv Drug Deliv Rev 2015;86:61-71.

27. Klein K, Winter S, Turpeinen M, et al. Pathway-Targeted Pharmacogenomics of CYP1A2 in Human Liver. Front Pharmacol 2010;1:129.

28. du Souich P, Fradette C. The effect and clinical consequences of hypoxia on cytochrome P450, membrane carrier proteins activity and expression. Expert Opin Drug Metab Toxicol 2011;7:1083-100.

29. Laybourn C, Tonnesen P, Loft S, et al. Pulmonary disease and antipyrine clearance. Clin Pharmacol Ther 1986;40:415-9.

30. Lainscak M, Vitale C, Seferovic P, et al. Pharmacokinetics and pharmacodynamics of cardiovascular drugs in chronic heart failure. Int J Cardiol 2016;224:191-8.

31. Richer M, Lam YW. Hypoxia, arterial $\mathrm{pH}$ and theophylline disposition. Clin Pharmacokinet 1993;25:283-99.

32. Gola S, Gupta A, Keshri GK, et al. Evaluation of hepatic metabolism and pharmacokinetics of ibuprofen in rats under chronic hypobaric hypoxia for targeted therapy at high altitude. J Pharm Biomed Anal 2016;121:114-22.

33. Legendre C, Hori T, Loyer P, et al. Drug-metabolising enzymes are down-regulated by hypoxia in differentiated human hepatoma HepaRG cells: HIF-1alpha involvement in CYP3A4 repression. Eur J Cancer 2009;45:2882-92.

34. da Rosa DP, Forgiarini LF, e Silva MB, et al. Antioxidants inhibit the inflammatory and apoptotic processes in an intermittent hypoxia model of sleep apnea. Inflamm Res 2015;64:21-9.

35. Ding W, Zhang Q, Dong Y, et al. Adiponectin protects the rats liver against chronic intermittent hypoxia induced injury through AMP-activated protein kinase pathway. Sci Rep 2016;6:34151.

36. Wei Q, Bian Y, Yu F, et al. Chronic intermittent hypoxia induces cardiac inflammation and dysfunction in a rat obstructive sleep apnea model. J Biomed Res 2016;30:490-5.

37. Wang Y, Hai B, Niu X, et al. Chronic intermittent hypoxia disturbs insulin secretion and causes pancreatic injury via MAPK signaling pathway. Biochem Cell Biol 2017;95:415-20.

38. Padmini E, Tharani J. Differential expression of HO-1 and CYP1A2 during up-regulation of ERK in stressed fish hepatocytes. Environ Monit Assess 2015;187:4147.

39. Tomankova V, Liskova B, Skalova L, et al. Altered cytochrome $\mathrm{P} 450$ activities and expression levels in the liver and intestines of the monosodium glutamate-induced mouse model of human obesity. Life Sci 2015;133:15-20.

40. Tabur S, Oztuzcu S, Oguz E, et al. CYP gene expressions in obesity-associated metabolic syndrome. Obes Res Clin Pract 2016;10:719-23.
Cite this article as: Zhang $\mathrm{XB}$, Zeng YM, Chen XY, Zhang YX, Ding JZ, Xue C. Decreased expression of hepatic cytochrome P450 1A2 (CYP1A2) in a chronic intermittent hypoxia mouse model. J Thorac Dis 2018;10(2):825-834. doi: $10.21037 /$ jtd.2017.12.106 